\title{
Integration of genetic and epigenetic markers for risk stratification: opportunities and challenges
}

\section{"Combining genetic and epigenetic markers provides huge potentials for risk stratification in cancer control programs."}

First draft submitted: 13 November 2015; Accepted for publication: 24 November 2015; Published online: 10 February 2016

Keywords: cancer • epigenetics • polygenic risk • risk assessment • risk stratification

To date, model-based estimates have shown the potential utility of common genetic susceptibility loci for risk stratification in cancer prevention and screening programs at population level $[1,2]$. A risk stratified screening strategy has the potential to improve the efficiency of the screening program and reduce its adverse consequences. For example, a risk-stratified screening strategy for breast cancer with eligibility for screening based on an absolute risk that is dependent on age and polygenic risk profile has been shown to reduce the number of women invited to screening while detecting most cancers potentially detectable by a conventional agebased screening strategy [1]. Studies in prostate cancer have shown that targeting screening to men at higher than population average risk, based on polygenic risk, could reduce the proportion of prostate cancers likely to be overdiagnosed [3,4].

A risk-stratified prevention or screening program would involve risk assessment, then stratifying the population into several risk groups and offering the intervention differentially to each population stratum with the expectation of improving the benefit-harm balance of the intervention [5]. Interventions, like screening, would be risk-tailored, with varying start and end age, frequency and modality of screening [6].

For risk assessment, genetic and environmental risk factors could be used. A broad definition of environment includes lifestyle, nutrition, external environmental exposures and reproductive history [7]. To date, genomewide association studies have identified 94 breast cancer susceptibility variants [8]. The polygenic risk profile based on these variants has area under the receiver operator characteristic curve $\left(\mathrm{AUC}_{-\mathrm{ROC}}\right)$ of 0.65 . AUC $_{\text {-ROC }}$ is the probability that a test correctly identifies an individual who will develop the disease from a pair of whom one will be affected and one will remain unaffected. AUC $_{- \text {ROC }}$ values range from 0.5 (total lack of discrimination) to 1.0 (perfect discrimination) [9]. Combining polygenic risk with environmental risk factors, like age of menarche, number of births, age of first live birth, oral contraceptive use, BMI, alcohol, smoking, personal history of benign breast disease and family history of breast cancer in first-degree relatives [2], would increase $\mathrm{AUC}_{-\mathrm{ROC}}$ to 0.68 . Even a modest increase in AUC ${ }_{\text {-ROC }}$ following combining genetic with environmental risk factors would substantially improve risk stratification [2].

\section{Epigenome as readout for environmental exposures}

Age, environmental exposures (such as tobacco, alcohol, infectious agents) and endogenous stimuli (such as circulating hormones) can trigger alterations in the epigenetic pattern. These alterations affect gene expression without changing the nucleotide sequence. Epigenetic changes are generally stable and propagate over cell divisions resulting in changes in phenotype. The pre-

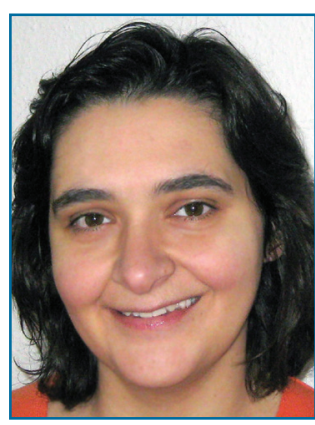

Nora Pashayan

Author for correspondence: Department of Applied Health Research, University College London, 1-19 Torrington Place, London, WC1E 7HB, UK

Tel: +44 2031083255

Fax: +44 2078130280

n.pashayan@ucl.ac.uk

\section{Daniel Reisel}

Department of Women's Cancer, University College London, London, WC1E 6BT, UK

Martin Widschwendter Department of Women's Cancer, University College London, London, WC1E 6BT, UK 
dominant mechanisms that may act alone or in combination to regulate gene expression over the lifetime of an organism include DNA methylation, histone modification, miRNA expression and processing, and chromatin condensation [10].

Epigenetic mechanisms are implicated in the initiation and progression of cancer through tumor suppressor genes silencing and/or oncogenes activation. DNA methylation is the most studied mechanism of epigenetic gene regulation [11]. DNA methylation refers to the addition of methyl group to cytosine base that is located $5^{\prime}$ to a guanosine base in a CpG dinucleotide. Regions of the genome rich in $\mathrm{CpG}$ are known as $\mathrm{CpG}$ islands, which are found in the promotor region of approximately half of all genes. Aberrant promotor hypermethylation associated with inappropriate gene silencing plays important role in tumor progression [12], whereas genome-wide decrease in methylation may lead to genomic instability and is associated with tumor progression [13].

\section{"...it is important to differentiate between association and causality of epigenetic mark with disease."}

Environmental exposures may promote tumor development by inducing both epigenetic and genetic changes (like mutations). The epigenetic makeup may play a role in the cellular response to these environmental exposures. Epigenetics may mediate or modify genetic risk [14]. As such individual's susceptibility to cancer would depend on both genetics and epigenetics makeup [15].

\section{Opportunities \& challenges}

Epigenetic changes unlike the genetic ones are reversible and can be modulated, for example, by diet, drugs and other environmental factors. This reversibility provides opportunity for cancer prevention strategies [10].

Environmentally triggered epigenetic changes are acquired gradually and influence tumor initiation and progression. In cervical cancer, epigenetic changes have been detected in morphologically normal cells, years in advance of neoplastic transformation [16]. DNA methylation has been shown to be differentially variable between normal, intraepithelial neoplasia and invasive cervical cancers [17]. In esophageal cancer, DNA methylation profile has been shown to predict progression of dysplasia in Barrett's esophagus [18]. These indicate that epigenetic changes could be used for risk prediction and early diagnosis.

Epigenetic alterations can act as surrogate markers of environmental exposures during individual's lifetime. Information collected on environmental exposures via questionnaire or direct measurement is susceptible to recall bias and to inadequate capturing of exposures with short half-lives and of low biological dose. Epigenetic markers when used in lieu could overcome some of these limitations [14]. As combining polygenic risk with environmental risk factors improves risk stratification, then integration of epigenetic markers and polygenic risk into a risk assessment tool would potentially improve risk stratification in cancer screening and prevention programs.

Unlike the genome, which is the same for all types of cells, the epigenome varies across cell types, in the same cell at different developmental stage, under the influence of different environmental exposures, and over time. For example, buccal cells exhibit significantly more smoking associated DNA methylation changes than blood cells. These changes correlate with DNA methylation changes in epithelial cancers and particularly with smoke-related epithelial cancers, notably lung cancer [19]. The tissue specificity of the epigenetic changes raises an issue with accessing tissue samples from organs like the prostate, ovary, breast, which are inaccessible by noninvasive means. Alternatively, tissue surrogates could be used. For example, epithelial cells from the uterine cervix are hormone sensitive and are likely to capture an 'epigenetic record' of breast cancer risk factors, and hence could be used as surrogate for breast cancer cells [20].

This plasticity of the epigenome poses several challenges in epigenetic testing. While polygenic risk could be determined at any point in time, a risk score that includes epigenetic markers needs to be evaluated several times to follow cancer-associated alterations. To identify a risk marker, it is important to differentiate between association and causality of epigenetic mark with disease [10], that is whether a change is adaptive response to environmental exposures or adverse effect with phenotypic consequences [15]. International efforts, such as the International Human Epigenome Consortium, and the reference epigenome, will enable comparison and identification of adverse effects [15].

Integration of different types of genomic, epigenomic and epidemiological data would be challenging. To overcome this, there is need for engaging geneticists, bioinformaticians, statisticians and clinicians and for developing improved strategies for handling large databases, data analysis and interpretation.

\section{Future perspective}

The European Commission Framework Programmes, Horizon 2020, recently funded the multidisciplinary research program, female cancer prediction using cervical omics to individualize screening and prevention (FORECEE) [21] to assess the validity, public health utility, cost-effectiveness, acceptabil- 
ity, and ethical, legal, social and regulatory impacts of using genomics, epigenomics and metagenomics data in personalized screening and prevention programs for breast, ovarian, endometrial and cervical cancers. To discover new DNA methylation signatures, an epigenome-wide association study will be conducted using prospectively collected cervical cells from liquid-based cytology cervical smears. Women's cancer risk identification test that combines DNA methylation signatures with SNPs and the microbiome will be developed and validated to predict the absolute risk of developing breast, ovarian, endometrial or cervical cancer. In the coming 4 years, the multidisciplinary team with expertise in oncology, genetics, genetics statistics, omics technologies, bioinformatics, decision analysis, epidemiology, public health, health economics, ethics and risk communication, and will be addressing the challenges with

\section{References}

1 Pashayan N, Duffy SW, Chowdhury S et al. Polygenic susceptibility to prostate and breast cancer: implications for personalised screening. Br. J. Cancer 104(10), 1656-1663 (2011).

2 Garcia-Closas M, Gunsoy NB, Chatterjee N. Combined associations of genetic and environmental risk factors: implications for prevention of breast cancer. J. Natl Cancer Inst. 106(11), 1-6 (2014).

3 Pashayan N, Duffy SW, Neal DE et al. Implications of polygenic risk-stratified screening for prostate cancer on overdiagnosis. Genet. Med. 17(10), 789-795 (2015).

4 Pashayan N, Pharoah PD, Schleutker J, Talala K, Tammela TL, Maattanen L et al. Reducing overdiagnosis by polygenic risk-stratified screening: findings from the Finnish section of the ERSPC. Br. J. Cancer 113, 1086-1093 (2015).

5 Burton H, Sagoo GS, Pharoah P, Zimmern RL. Time to revisit Geoffrey Rose: strategies for prevention in the genomic era? Ital. J. Public Health 9(4), e8665-1-e8665-9 (2012).

6 Dent T, Jbilou J, Rafi I et al. Stratified cancer screening: the practicalities of implementation. Public Health Genomics 16(3), 94-99 (2013).

7 Widschwendter M, Jones A, Teschendorff AE. Epigenetics makes its mark on women-specific cancers - an opportunity to redefine oncological approaches? Gynecol. Oncol. 128(1), 134-143 (2013).

8 Michailidou K, Beesley J, Lindstrom S et al. Genome-wide association analysis of more than 120,000 individuals identifies 15 new susceptibility loci for breast cancer. Nat. Genet. 47(4), 373-380 (2015).

9 van ZM, van der Net JB, Kundu S, Freedman AN, van Duijn $\mathrm{CM}$, Janssens AC. Genome-based prediction of breast cancer risk in the general population: a modeling study based on metaanalyses of genetic associations. Cancer Epidemiol. Biomarkers Prev. 20(1), 9-22 (2011).

10 Verma M, Khoury MJ, Ioannidis JP. Opportunities and challenges for selected emerging technologies in cancer developing and implementing omics-based cancer prevention and screening program.

\section{Conclusion}

Cancer is a genetic and epigenetic disease. Combining genetic and epigenetic markers provides huge potentials for risk stratification in cancer control programs. Multidisciplinary efforts are needed to overcome the challenges.

\section{Financial \& competing interests disclosure}

N Pashayan is a Cancer Research UK Clinician Scientist Fellow. The authors have no other relevant affiliations or financial involvement with any organization or entity with a financial interest in or financial conflict with the subject matter or materials discussed in the manuscript apart from those disclosed.

No writing assistance was utilized in the production of this manuscript.

epidemiology: mitochondrial, epigenomic, metabolomic, and telomerase profiling. Cancer Epidemiol. Biomarkers Prev. 22(2), 189-200 (2013).

11 Widschwendter M, Jones PA. DNA methylation and breast carcinogenesis. Oncogene 21(35), 5462-5482 (2002).

12 Jones PA, Baylin SB. The fundamental role of epigenetic events in cancer. Nat. Rev. Genet. 3(6), 415-428 (2002).

13 Hamilton JP. Epigenetics: principles and practice. Dig. Dis. 29(2), 130-135 (2011).

14 Ladd-Acosta C, Fallin MD. The role of epigenetics in genetic and environmental epidemiology. Epigenomics doi:10.2217/ epi.15.102 (2015) (Epub ahead of print).

15 Herceg Z, Lambert MP, van Veldhoven K et al. Towards incorporating epigenetic mechanisms into carcinogen identification and evaluation. Carcinogenesis 34(9), 1955-1967 (2013).

16 Teschendorff AE, Jones A, Fiegl $\mathrm{H}$ et al. Epigenetic variability in cells of normal cytology is associated with the risk of future morphological transformation. Genome Med. 4(3), 24 (2012).

17 Teschendorff AE, Liu X, Caren $\mathrm{H}$ et al. The dynamics of DNA methylation covariation patterns in carcinogenesis. PLoS Comput. Biol. 10(7), e1003709 (2014).

18 Jin Z, Cheng Y, Gu W et al. A multicenter, double-blinded validation study of methylation biomarkers for progression prediction in Barrett's esophagus. Cancer Res. 69(10), 4112-4115 (2009).

19 Teschendorff AE, Yang Z, Wong A et al. Correlation of smoking-associated DNA methylation changes in buccal cells with DNA methylation changes in epithelial cancer. JAMA Oncol. 1(4), 476-485 (2015).

20 Anjum S, Fourkala EO, Zikan M et al. A BRCA1-mutation associated DNA methylation signature in blood cells predicts sporadic breast cancer incidence and survival. Genome Med. 6(6), 47 (2014).

21 Cordis. Community Research and Development Information Service.

cordis.europa.eu/search/result_en?q=forecee 